\title{
Dynamic handgrip exercise for the evaluation of mitral valve regurgitation: an echocardiographic study to identify exertion induced severe mitral regurgitation
}

\author{
Mhd Nawar Alachkar ${ }^{1}$ (D) . Annemarie Kirschfink ${ }^{1} \cdot$ Julian Grebe ${ }^{1} \cdot$ Mohammad Almalla $^{1} \cdot$ Michael Frick $^{1}$. \\ Andrea Milzi ${ }^{1} \cdot$ Wiebke Moersen ${ }^{2} \cdot$ Michael Becker $^{2} \cdot$ Nikolaus Marx $^{1} \cdot$ Ertunc Altiok $^{1}$
}

Received: 8 June 2020 / Accepted: 5 October 2020 / Published online: 16 October 2020

(c) The Author(s) 2020

\begin{abstract}
Handgrip exercise (HG) has been occasionally used as a stress test in echocardiography. The effect of HG on mitral regurgitation (MR) is not well known. This study aims to evaluate this effect and the possible role of HG in the echocardiographic evaluation of MR. 722 patients with MR were included (18\% primary, $82 \%$ secondary disease). We calculated effective regurgitant orifice area (EROA) and regurgitant volume (RVOL) at rest and during dynamic HG. Increase in MR was defined as any increase in EROA or RVOL. We analyzed the data to identify possible associations between clinical or echocardiographic parameters and the effect of $\mathrm{HG}$ on MR. MR increased during dynamic HG in 390 of 722 patients $(54 \%)(\Delta \mathrm{EROA}=25 \%$, $\triangle \mathrm{RVOL}=27 \%)$. Increase of regurgitation occurred in 66 of 132 patients with primary MR (50\%) and in 324 of 580 patients with secondary MR (55\%). This increase was associated with larger baseline EROA and RVOL, but it was independent from other clinical or echocardiographic parameters. In secondary MR, dynamic HG led to a reclassification of regurgitation severity from non-severe at rest to severe MR during HG in 104 of 375 patients (28\%). There was a significant association between this upgrade in MR classification and higher New York Heart Association (NYHA) class (OR 1.486, 95\%-CI 1.138-1.940, $\mathrm{p}=0.004$ ). Dynamic HG exercise increases MR in about half of patients independent of the etiology. Dynamic HG may be used to identify symptomatic patients with non-severe secondary MR at rest but severe MR during exercise.
\end{abstract}

Keywords Handgrip · Mitral valve regurgitation · Echocardiography

\begin{tabular}{ll}
\multicolumn{2}{l}{ Abbreviations } \\
EROA & Effective regurgitant orifice area \\
HG & Handgrip \\
HR & Heart rate \\
LA & Left atrial \\
LV-EF & Left ventricular ejection fraction \\
LVEDd & Left ventricular end-diastolic diameter \\
LVEDs & Left ventricular end-systolic diameter \\
MR & Mitral regurgitation \\
NT-proBNP & NT-pro brain natriuretic peptide \\
NYHA & New York Heart Association \\
PISA & Proximal isovelocity surface area
\end{tabular}

Mhd Nawar Alachkar

nalachkar@ukaachen.de

1 Department of Cardiology, Angiology and Intensive Care, University Hospital RWTH Aachen, Aachen, Germany

2 Department of Cardiology, Nephrology and Intensive Care, Rhein-Maas Hospital, Wuerselen, Aachen, Germany

$\begin{array}{ll}\text { RVOL } & \text { Regurgitant volume } \\ \text { SPAP } & \text { Systolic pulmonary artery pressure } \\ \text { TAPSE } & \text { Tricuspid annular plane systolic excursion } \\ \text { TTE } & \text { Transthoracic echocardiograp }\end{array}$

\section{Background}

Handgrip exercise (HG) is a simple bedside maneuver to increase the intensity of murmur of mitral regurgitation (MR) during auscultation [1]. In addition, $\mathrm{HG}$ has been used in echocardiography as a means of stress test to identify exertion induced ischemia or exertion induced left ventricular diastolic dysfunction [2, 3]. Furthermore, HG has been used in combination with dobutamine stress echocardiography to identify patients with MR who would probably benefit from percutaneous mitral valve repair [4]. However, there is no existing data describing the effect of isolated HG on the severity of MR. 
MR is the most common valvular heart disease and represents the second most common indication for valvular heart surgery $[5,6]$. In patients with heart failure, MR is associated with an adverse prognosis [7]. Therapeutic options are increasing due to evolving of transcatheter techniques for MR treatment $[8,8]$. Therefore, a precise evaluation of the impact of MR on clinical symptoms is essential. Exercise echocardiography has been described to improve assessment of MR severity [9, 10]. It may also be used to predict outcome in patients with heart failure and mitral regurgitation, as a large exercise-induced increase in MR is associated with higher morbidity and mortality [11]. However, performing an echocardiographic study during dynamic exercise such as running on a treadmill or cycling on ergometer may not always be feasible $[12,13]$. The effect of HG exercise as an easy to perform method has not yet been systematically evaluated in patients with MR.

In this single-center study, we examined the effect of $\mathrm{HG}$ on MR in a large cohort of patients with different pathologies and severities of the disease. We aimed to investigate a possible role of $\mathrm{HG}$ in the echocardiographic evaluation of MR.

\section{Methods}

\section{Study population}

During the recruiting period, we screened all patients who received transthoracic echocardiography (TTE) in our laboratory. Every patient who was found to have MR, aside of its pathology or severity, and was sufficiently able to perform dynamic HG exercise was included. After completing the echocardiographic study at rest, the patient was requested to perform dynamic HG exercise. During the exercise, images of mitral regurgitation were acquired and saved for later evaluation. Due to the difficulty in applying the proximal isovelocity surface area (PISA) method for MR quantification in patients with a previous intervention on the mitral valve (percutaneous or surgical), those patients were excluded from the final analysis. Furthermore, patients in whom the use of PISA method for MR quantification was not suitable (e.g. very eccentric jet or multiple jets) were also excluded. Finally, we included 722 patients with different severities of MR.

\section{Transthoracic echocardiography}

Echocardiographic studies were performed with patients lying in left decubitus position. Examinations were performed using commercially available echocardiographic system (Vivid E9. General Electric Vingmed. Horten. Norway) and 2D transthoracic probe (M5S. General Electric Vingmed. Horten. Norway). Echocardiographic examinations were performed according to recommended standards of the European association of echocardiography [14]. In patients with atrial fibrillation, all echocardiographic measurements were done over at least three cycles and the average value of these measurements was determined to minimize variability. Apical 4-chamber view was used for calculation of effective regurgitant orifice area (EROA) and the regurgitant volume (RVOL) using the PISA method. Maximal instantaneous regurgitant flow was calculated as $2 * \pi * r^{2} *$ $\mathrm{v}_{\text {aliasing, }}$, where $\mathrm{r}$ was the maximal distance to the contour of aliasing velocity $\mathrm{v}_{\text {aliasing }}$ with a hemispheric contour assumed. EROA was obtained by dividing maximal instantaneous regurgitant flow by peak regurgitant velocity obtained by continuouswave Doppler [15]. We calculated EROA and RVOL of MR at rest and during $\mathrm{HG}$. Increase of MR was defined as any increase in EROA or RVOL during dynamic $\mathrm{HG}$ exercise.

Other echocardiographic parameters such as left ventricular ejection fraction (LV-EF), left atrial area (LA), systolic pulmonary artery pressure (sPAP) and tricuspid annular plane systolic excursion (TAPSE) were calculated. The association between these parameters and the effect of HG on MR was assessed.

\section{Handgrip technique}

After completing the echocardiographic examination at rest, patients were given a small, elastic, hand-sized training ball in each hand and they were instructed to perform dynamic HG exercise while still lying in left decubitus position. Patients were advised to contract and open their hands in a continuous manner to squeeze and release the ball repeatedly. The exercise was regularly continued for $3 \mathrm{~min}$.

\section{Additional information}

Clinical characteristics of the patients including coronary artery disease, New York Heart Association (NYHA) dyspnea classification, the presence of current or any history of atrial fibrillation were obtained from the previous medical 
documents of the patients. Unless clinically indicated, coronary artery disease was not newly investigated in the context of this study. These characteristics were assessed to evaluate a possible association between these factors and the effect of dynamic HG on MR. NT-pro brain natriuretic peptide (NT-proBNP) level was also evaluated to identify a possible association between heart failure and the increase of MR during dynamic $\mathrm{HG}[16,17]$.

\section{Data analysis}

Clinical and echocardiographic data of the patients were retrospectively analyzed. We classified patients according to the etiology of disease into primary and secondary MR [18]. Analysis was carried out in all patients and in each of the two groups separately. Finally, to investigate if $\mathrm{HG}$ exercise would lead to an upgrade in classification of regurgitation severity from non-severe MR at rest to severe MR during HG, we performed an additional analysis. For this analysis, patients who already had severe MR at rest (patients with EROA $\geq 40 \mathrm{~mm}^{2}, \mathrm{RVOL} \geq 60 \mathrm{ml}$ in primary MR and $E R O A \geq 20 \mathrm{~mm}^{2}, R V O L \geq 30 \mathrm{ml}$ in secondary MR) were excluded $[15,19]$.

\section{Statistical analysis}

All statistical analysis was performed with SPSS software (version 25, IBM Corp., Armonk, NY, USA) and MedCalc software (version 19.1; Mariakerke, Belgium).

Categorical variables were summarized as count (percentage) and continuous variables as mean \pm standard deviation. Distributions of continuous variables were compared with t-test, association of categorical variables was assessed by Pearson's chi-square test. To find variables for prediction of the increase in MR under HG univariate logistic regression analysis was performed. $\mathrm{p}$ values $<0.05$ were considered significant.

\section{Results}

Clinical characteristics and echocardiographic findings of the patients are summarized in Table 1. Among these patients, 132 were classified as primary MR (18\%) and 590 as secondary MR (82\%).

\section{Effect of handgrip on heart rate.}

During dynamic HG exercise, mean heart rate (HR) increased from $72 \pm 12 / \mathrm{min}$ to $78 \pm 13 / \mathrm{min}$. HR increased during HG in 527 patients (73\%) while in 195 patients (27\%) no increase of HR was observed. In patients with primary MR, HR increased from $69 \pm 10 /$ min to $76 \pm 13$ / $\min (\Delta \mathrm{HR}=10.1 \%)$. In patients with secondary MR, HR increased from $72 \pm 12 / \mathrm{min}$ to $78 \pm 14 / \mathrm{min}(\Delta \mathrm{HR}=8.3 \%)$ (Table 1).

\section{Effect of handgrip on the severity of MR.}

An increase in MR during HG exercise, defined as any increase in EROA or RVOL, occurred in 390 patients (54\%) (Fig. 1). In these patients, EROA increased by $4 \pm 6 \mathrm{~mm}^{2}$ ( $25 \%$ of the baseline value) and RVOL by $7 \pm 9 \mathrm{ml}(27 \%$ of the baseline value). This increase was significantly associated with a larger baseline EROA at rest (OR 1.027, 95\%-CI $1.008-1.045, \mathrm{p}=0.004)$ and with a greater baseline RVOL at rest (OR 1.015, 95\%-CI 1.003-1.027, $\mathrm{p}=0.012$ ). It was also significantly associated with LA area (OR $1.025,95 \%$-CI $1.005-1.045, p=0.014)$. In contrast, there was no association between the increase of MR during HG and any other echocardiographic parameters including LV dimensions or LV-EF. There was no association between this increase and clinical parameters including NYHA dyspnea class, NTproBNP or atrial fibrillation (Table 2).

\section{Differentiation between primary and secondary MR.}

We classified the patients in two groups based on the etiology of MR. HG exercise increased regurgitation severity in 50\% of patients with primary MR, and in 55\% with secondary MR. The extent of this increase was not significantly different between patients with secondary MR $\left(\triangle \mathrm{EROA}=4 \pm 6 \mathrm{~mm}^{2}, 26 \%\right.$ of baseline value; $\Delta \mathrm{RVOL}=7 \pm 9 \mathrm{ml}, 28 \%$ of baseline value) and those with primary MR $\left(\triangle \mathrm{EROA}=3.5 \pm 5 \mathrm{~mm}^{2}, 20 \%\right.$ of baseline value; $\Delta \mathrm{RVOL}=6.5 \pm 9 \mathrm{ml}, 22 \%$ of baseline value $)(\mathrm{p}=0.261$ for $\triangle E R O A$ and $\mathrm{p}=0.426$ for $\triangle \mathrm{RVOL}$; respectively). There was an association between this increase and LA size in patients with primary MR (OR 1.025, 95\%-CI 1.005-1.945, $\mathrm{p}=0.040$ ) but not in those with secondary MR (OR 1.018, $95 \%$-CI $0.997-1.040, \mathrm{p}=0.093)$. There was a significant association between the increase of MR during HG and baseline EROA and baseline RVOL at rest in patients with secondary (OR 1.038, 95\%-CI 1.015-1.061, $\mathrm{p}=0.001$ and OR $1.01595 \%$-CI $1.001-1.028, \mathrm{p}=0.032$; respectively) but not in those with primary MR (OR $1.008,95 \%$-CI $0.980-1.036, p=0.593$ and OR $1.018,95 \%$-CI $0.994-1.042$, $\mathrm{p}=0137$; respectively). However, there was no association between this increase of MR during HG and any other echocardiographic parameters including LV dimensions or LV-EF. Furthermore, there was no association between this increase and clinical parameters including NYHA dyspnea class, NT-proBNP or atrial fibrillation in any of the two groups (Fig. 2, Table 3). 
Table 1 Clinical characteristics and echocardiographic parameters.

\begin{tabular}{|c|c|c|c|c|}
\hline Variable & $\begin{array}{l}\text { All patients, } \\
\mathrm{n}=722\end{array}$ & $\begin{array}{l}\text { Primary MR, } \\
n=132\end{array}$ & $\begin{array}{l}\text { Secondary MR, } \\
\mathrm{n}=590\end{array}$ & $\mathrm{p}$-value \\
\hline \multicolumn{5}{|l|}{ Clinical characteristics } \\
\hline Age, years & $72 \pm 12$ & $68 \pm 16$ & $73 \pm 10$ & 0.001 \\
\hline Men, $\mathrm{n}$ & $380(52 \%)$ & $44(34 \%)$ & $335(57 \%)$ & $<0.001$ \\
\hline $\mathrm{CAD}, \mathrm{n}$ & $490(68 \%)$ & $24(18 \%)$ & $466(79 \%)$ & $<0.001$ \\
\hline Atrial fibrillation, $\mathrm{n}$ & $412(57 \%)$ & $63(48 \%)$ & $348(59 \%)$ & 0.051 \\
\hline NT-proBNP, pg/ml & $3347 \pm 3696$ & $1275 \pm 1310$ & $3660 \pm 3837$ & $<0.001$ \\
\hline Hemoglobin, mg/dl & $12.3 \pm 1.8$ & $13.4 \pm 1.7$ & $12 \pm 1.8$ & 0.025 \\
\hline Creatinine, $\mathrm{mg} / \mathrm{dl}$ & $1.3 \pm 0.8$ & $0.95 \pm 0.2$ & $1.5 \pm 0.9$ & $<0.001$ \\
\hline NYHA class & & & & $<0.001$ \\
\hline I & $188(26 \%)$ & $59(45 \%)$ & $129(22 \%)$ & \\
\hline II & $223(31 \%)$ & $36(27 \%)$ & $188(32 \%)$ & \\
\hline III & $271(38 \%)$ & $32(24 \%)$ & $241(41 \%)$ & \\
\hline IV & $37(5 \%)$ & $6(4 \%)$ & $32(5 \%)$ & \\
\hline Mean NYHA class & 2.2 & 1.8 & 2.3 & 0.01 \\
\hline Beta-blocker, $\mathrm{n}$ & $613(85 \%)$ & $91(70 \%)$ & $521(88 \%)$ & 0.01 \\
\hline Presence of $\mathrm{HR}$ increase during $\mathrm{HG}, \mathrm{n}$ & $527(73 \%)$ & $103(78 \%)$ & $432(72 \%)$ & 0.225 \\
\hline Mean increase of HR during HG, beat/min & $6 \pm 8$ & $7 \pm 8$ & $6 \pm 8$ & 0.118 \\
\hline \multicolumn{5}{|l|}{ Echocardiographic parameters } \\
\hline LV-EF, \% & $44 \pm 13$ & $58 \pm 6$ & $41 \pm 12$ & $<0.001$ \\
\hline LA area, $\mathrm{cm}^{2}$ & $26 \pm 7$ & $23 \pm 7$ & $26 \pm 8$ & $<0.001$ \\
\hline sPAP, mmHG & $35 \pm 12$ & $32 \pm 12$ & $36 \pm 11$ & 0.005 \\
\hline TAPSE, mm & $19 \pm 5$ & $21 \pm 4$ & $19 \pm 5$ & $<0.001$ \\
\hline EROA at rest, $\mathrm{mm}^{2}$ & $18 \pm 9$ & $18 \pm 12$ & $17 \pm 8$ & 0.324 \\
\hline EROA during $\mathrm{HG}, \mathrm{mm}^{2}$ & $22 \pm 11$ & $22 \pm 14$ & $22 \pm 10$ & 0.870 \\
\hline$\Delta \mathrm{EROA}, \mathrm{mm}^{2}$ & $4 \pm 6$ & $3.5 \pm 5$ & $4 \pm 6$ & 0.261 \\
\hline RVOL at rest, $\mathrm{ml}$ & $30 \pm 9$ & $31 \pm 15$ & $28 \pm 12$ & 0.260 \\
\hline RVOL during HG, ml & $37 \pm 17$ & $38 \pm 19$ & $37 \pm 16$ & 0.676 \\
\hline$\Delta \mathrm{RVOL}, \mathrm{ml}$ & $7 \pm 9$ & $6.5 \pm 9$ & $7 \pm 9$ & 0.426 \\
\hline LVEDd, mm & $53 \pm 9$ & $48 \pm 6$ & $54 \pm 10$ & $<0.001$ \\
\hline LVEDs, mm & $41 \pm 12$ & $33 \pm 7$ & $43 \pm 12$ & $<0.001$ \\
\hline
\end{tabular}

$C A D$ coronary artery disease

EROA effective regurgitant orifice area

$H G$ handgrip

$H R$ heart rate

$L A$ area left atrial area

$L V E D d$ left ventricular end-diastolic diameter

$L V E D$ s left ventricular end-systolic diameter

$L V$-EF left ventricular ejection fraction

NYHA New York Heart Association

$R V O L$ regurgitant volume

$S P A P$ systolic pulmonary artery pressure

TAPSE tricuspid annular plane systolic excursion
Effect of HG on the classification of MR severity.

In order to evaluate if $\mathrm{HG}$ exercise may lead to a reclassification of MR severity from non-severe MR at rest to severe MR during HG, we excluded patients who already had severe MR at rest for this additional analysis. In patients with primary disease, MR was classified as non-severe $\left(\mathrm{EROA}<40 \mathrm{~mm}^{2}, \mathrm{RVOL}<60 \mathrm{ml}\right)$ in 125 patients at rest. In 7 of these patients (5\%) there was an increase from nonsevere to severe MR (EROA $\geq 40 \mathrm{~mm}^{2}, \mathrm{RVOL} \geq 60 \mathrm{ml}$ ) during dynamic $\mathrm{HG}$ (Fig. 3). There was a significant association between baseline EROA and RVOL and this upgrade in the 

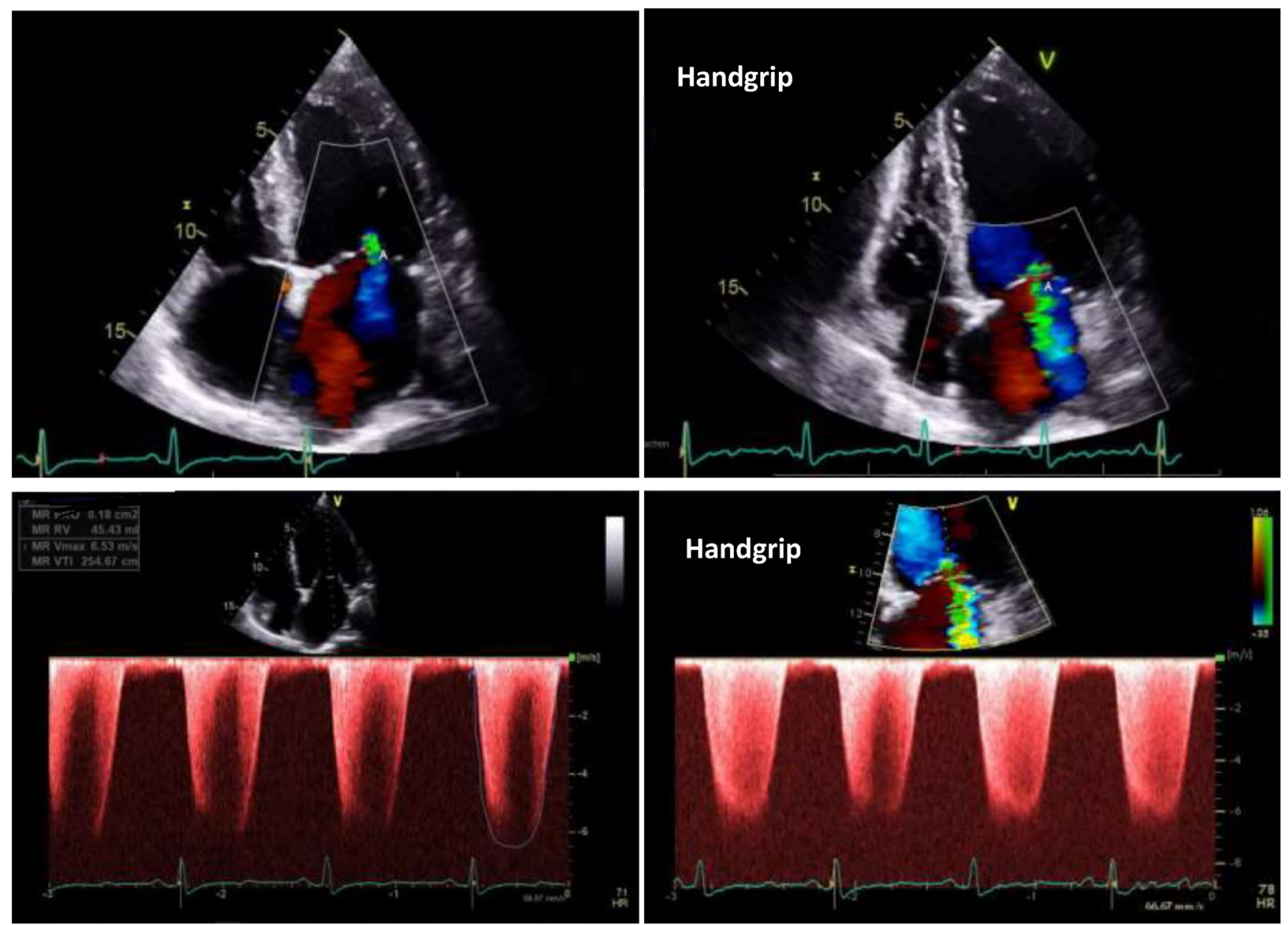

Fig. 1 Baseline echocardiographic apical 4-chamber view with color Doppler (upper left) with continuous-wave Doppler assessment (lower left) in a patient with secondary mitral regurgitation (MR).

There was an increase of MR (upper right) with denser continuouswave Doppler signal during handgrip exercise (lower right)

classification of MR severity during HG. However, there was no association between this upgrade in the classification of MR severity and other clinical or echocardiographic parameters in primary disease (Tables 4, 5).

In patients with secondary disease, MR was classified as non-severe (EROA $<20 \mathrm{~mm}^{2}, \mathrm{RVOL}<30 \mathrm{ml}$ ) in 375 patients at rest. In 104 of these patients (28\%) there was an increase from non-severe to severe MR (EROA $\geq 20 \mathrm{~cm}^{2}$, RVOL $\geq 30 \mathrm{ml}$ ) during HG (Fig. 3). There was a significant association between baseline EROA and RVOL and this upgrade in the classification of MR severity during HG. Furthermore, there was a significant association between the severity of exertional dyspnea (NYHA class) and this upgrade in the classification of MR severity (OR 1.486, 95\%-CI 1.138-1.940, $\mathrm{p}=0.004)$. Moreover, intake of betablocker was more common in patients with upgrade in

the classification of MR severity than in patients without this increase ( $94 \%$ vs $86 \%$; $=0.026$ ). There was no association between this upgrade in the classification of MR severity and any other clinical or echocardiographic parameters in secondary disease (Tables 4,5 ).

\section{Discussion}

The major findings of this study are (1) dynamic HG exercise increases MR in about half of patients independent of the etiology, (2) the absolute increase of MR during HG is significantly associated with larger baseline EROA and RVOL in patients with secondary MR and with more dilated LA in those with primary MR, (3) dynamic HG exercise changes the classification of MR severity from non-severe 
Table 2 Association between clinical variables and echocardiographic parameters and the increase of MR during $\mathrm{HG}$ in the study population

\begin{tabular}{lll}
\hline Variable & OR, 95\%-CI & p-value \\
\hline Clinical variables & & \\
Age & $1.008(0.966-1.020)$ & 0.211 \\
Gender & $0.920(0.686-1.233)$ & 0.576 \\
Atrial fibrillation & $0.988(0.735-1.327)$ & 0.934 \\
NYHA functional classification & $1.104(0.936-1.302)$ & 0.239 \\
(per class) & & \\
NT-proBNP & $1.000(1.000-1.000)$ & 0.442 \\
HR at rest & $1.000(0.989-1.012)$ & 0.949 \\
HR during HG & $1.002(0.991-1.013)$ & 0.710 \\
Increase of HR during HG & $1.005(0.298-4.358)$ & 0.849 \\
Beta-blocker & $1.774(1.170-2.688)$ & 0.218 \\
Echocardiographic parameters & & \\
EROA at rest & $1.027(1.008-1.045)$ & 0.004 \\
RVOL at rest & $1.015(1.003-1.027)$ & 0.012 \\
LV-EF & $0.995(0.985-1.006)$ & 0.411 \\
LA area & $1.025(1.005-1.945)$ & 0.014 \\
sPAP & $1.002(0.989-1.015)$ & 0.801 \\
TAPSE & $0.996(0.968-1.024)$ & 0.760 \\
LVEDd & $1.001(0.995-1.027)$ & 0.176 \\
LVEDs & $1.006(0.991-1.023)$ & 0.330 \\
\hline EROA & &
\end{tabular}

$E R O A$ effective regurgitant orifice area

$L A$ area left atrial area

LVEDd left ventricular end-diastolic diameter

$L V E D$ s left ventricular end-systolic diameter

$L V$-EF left ventricular ejection fraction

NYHA New York Heart Association

RVOL regurgitant volume

$S P A P$ systolic pulmonary artery pressure

TAPSE tricuspid annular plane systolic excursion

MR at rest to severe MR during HG in about one third of patients with secondary MR with a significant association between this reclassification and the severity of exertional dyspnea in this subgroup.

General cardiovascular response to exercise has been described in detail [20]. However, this response may vary according to the type of exercise. Cardiovascular response to static exercise such as static handgrip or weightlifting is different from response to dynamic exercise such as swimming or running. Dynamic exercise causes a relevant increase in heart rate, cardiac output, and oxygen consumption with lesser effect on blood pressure. In contrast, isometric exercise causes mainly an increase in blood pressure with lesser effects on other parameters [21]. HG exercise is further divided into static, also known as sustained HG, where the hand is continuously and constantly contracted against a given resistance, and dynamic HG, also known as repeated hand-squeeze where the hand is contracted and opened continuously. Louhevaara et al. compared the cardiorespiratory response to each type of $\mathrm{HG}$ exercise and did not find any difference regarding blood pressure, heart rate or ventilatory gas exchange [23]. The extent of these hemodynamic effects of HG is determined by the intensity of the contraction and its duration [24]. In patients with primary MR, there is only limited data regarding the role of exercise echocardiography [25]. However, the effect of exercise on secondary MR is more investigated. Because the response to exercise in patients with cardiomyopathies is different from normal subjects, exercise leads to a volume and pressure overload on the left ventricle, causing its dilation and consequently an increase in secondary mitral regurgitation. This effect was observed in several studies [26]. Regarding static exercise, Keren and colleagues reported that static HG caused a significant increase in the severity of secondary MR in 17 patients with advanced heart failure [27]. However, this study was performed before the era of modern medical treatment or device-assisted heart failure therapy, which are known to improve MR in patients with heart failure [26]. The role of repeated hand-squeeze or dynamic handgrip exercise as a mix between static and dynamic exercise on MR is not known.

Our study showed that dynamic HG exercise increased mitral regurgitation in some patients. Nonetheless, this effect and its extent were extremely variable among the study population. Comparing these results to the study of Lancellotti et al., which examined the impact of dynamic exercise (bicycle exercise) on the severity of secondary MR, we saw a qualitatively similar, but quantitatively lesser effect of dynamic $\mathrm{HG}$ on MR compared to dynamic exercise [11]. In that study, a mean increase in EROA of $8 \pm 10 \mathrm{~mm}^{2}$ during exercise was noticed, compared to a mean increase in EROA of $4 \pm 6 \mathrm{~mm}^{2}$ during dynamic HG in our study. A variable effect of dynamic exercise on MR was also seen, as EROA decreased in some patients. Furthermore, our results showed that there was quantitatively more increase of regurgitation during $\mathrm{HG}$ in patients with more severe MR with larger baseline EROA and RVOL at rest, which was also comparable to the results from Lancellotti et al. [11], as EROA increased by $\geq 13 \mathrm{~mm}^{2}$ in 48 of 51 patients with severe secondary MR $\left(\right.$ EROA $\left.\geq 20 \mathrm{~mm}^{2}\right)$ at rest in their study. However, this comparison was only intended to give an idea about the similar effects of dynamic exercise and 

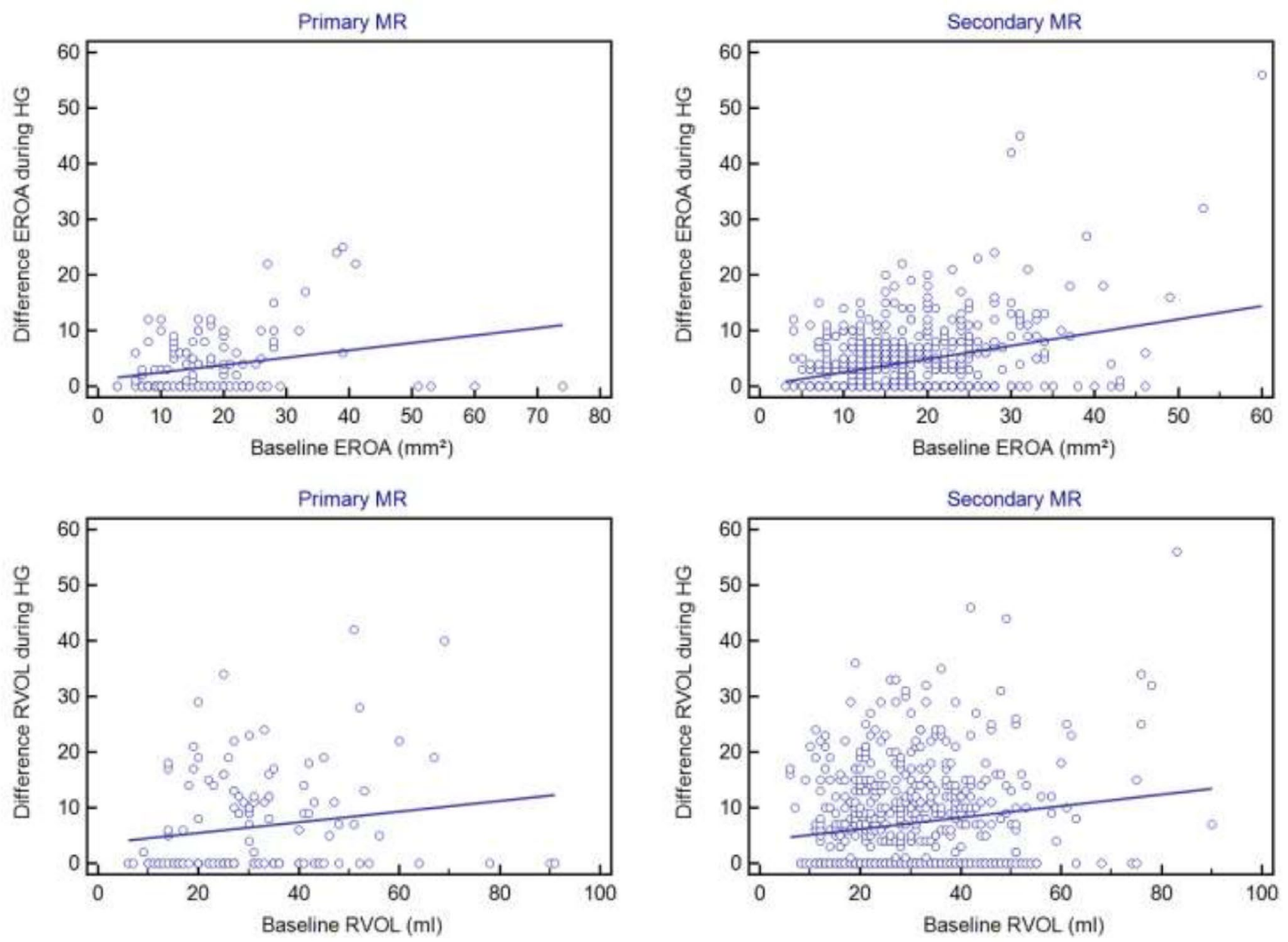

Fig. 2 Baseline effective regurgitant orifice area (EROA) and its increase during handgrip (HG) exercise in patients with primary (upper left) and secondary mitral regurgitation (MR) (upper right)

and corresponding baseline regurgitant volume (RVOL) and its increase during HG exercise in patients with primary (lower left) and secondary MR (lower right)

dynamic HG on MR and does not replace a head-to-head comparison of the two methods.

In clinical practice, the absolute increase of MR during HG may not play a major role but rather the exercise induced change in the classification of MR severity. According to the recent guidelines, severe MR at rest, in presence of other findings, is an indication for intervention [19]. But change in the classification of MR severity during dynamic HG may particularly be important in symptomatic patients with non-severe MR at rest. Our study showed that dynamic HG changed the classification of regurgitation severity from non-severe MR at rest to severe MR in about one third of patients with secondary MR. This effect was especially seen in symptomatic patients with exertional dyspnea and it was significantly associated with the severity of exertional dyspnea (NYHA class). This finding implies that dynamic

HG exercise during echocardiography may identify patients with non-severe secondary MR at rest, which may turn to severe MR during exertion. This is an important result and it may be the cornerstone for prospective studies assessing the prognostic importance of this finding and its role in deciding further management. The association between HG induced reclassification of MR severity and baseline EROA and RVOL is reasonable, as only less increase of these values is needed to upgrade the classification of regurgitation severity in patients with borderline MR at rest. As mentioned before, the effect of exercise on primary MR is not well established. Our results showed that dynamic HG had a similar effect on regurgitation in both primary and secondary MR, as MR increased by $20-28 \%$ in about half of the patients in both groups. However, in patients with primary MR, dynamic HG leaded to an upgrade in the classification in MR severity 
Table 3 Association between clinical variables and echocardiographic parameters and the increase in MR during HG in patients with primary and secondary MR

\begin{tabular}{|c|c|c|c|c|}
\hline \multirow[t]{2}{*}{ Variable } & \multicolumn{2}{|l|}{ Primary MR } & \multicolumn{2}{|l|}{ Secondary MR } \\
\hline & OR, $95 \%$-CI & p-value & OR, $95 \%$-CI & p-value \\
\hline \multicolumn{5}{|l|}{ Clinical variables } \\
\hline Age (per year) & $1.008(0.987-1.029)$ & 0.460 & $1.006(0.991-1.021)$ & 0.417 \\
\hline Gender (men) & $1.157(0.979-1.335)$ & 0.950 & $0.933(0.673-1.295)$ & 0.680 \\
\hline Atrial fibrillation & $1.095(0.552-2.173)$ & 0.796 & $0.953(0.685-1.326)$ & 0.775 \\
\hline NYHA functional Classification (per class) & $0.923(0.632-1.349)$ & 0.680 & $1.128(0.936-1.361)$ & 0.266 \\
\hline NT-proBNP & $1.000(1.000-1.000)$ & 0.872 & $1.000(1.000-1.000)$ & 0.450 \\
\hline Increase of heart rate during $\mathrm{HG}$ & $0.495(0.045-5.432)$ & 0.565 & $1.946(0.354-10.710)$ & 0.444 \\
\hline Beta-blocker & $2.033(0.949-4.354)$ & 0.066 & $1.610(0.965-2.686)$ & 0.068 \\
\hline \multicolumn{5}{|l|}{ Echocardiographic parameters } \\
\hline EROA at rest (per $\mathrm{mm}^{2}$ ) & $1.008(0.980-1.036)$ & 0.593 & $1.038(1.015-1.061)$ & 0.001 \\
\hline RVOL at rest (per ml) & $1.018(0.994-1.042)$ & 0.137 & 1.015 (1.001-1.028) & 0.032 \\
\hline LV-EF & $1.084(0.992-1.183)$ & 0.073 & $0.995(0.983-1.008)$ & 0.450 \\
\hline LA area $\left(\right.$ per $\left.\mathrm{cm}^{2}\right)$ & $1.056(1.003-1.113)$ & 0.040 & $1.018(0.997-1.040)$ & 0.093 \\
\hline sPAP (per mmHg) & $1.010(0.980-1.040)$ & 0.526 & $0.999(0.985-1.014)$ & 0.933 \\
\hline TAPSE (per mm) & $0.975(0.906-1.048)$ & 0.493 & $1.003(0.972-1.035)$ & 0.842 \\
\hline LVEDd (per mm) & $1.057(0.998-1.120)$ & 0.557 & $1.005(0.988-1.022)$ & 0.582 \\
\hline LVEDs (per mm) & $1.045(0.990-1.103)$ & 0.114 & $1.001(0.988-1.015)$ & 0.852 \\
\hline
\end{tabular}

EROA effective regurgitant orifice area

$L A$ area left atrial area

LVEDd left ventricular end-diastolic diameter

LVEDs left ventricular end-systolic diameter

$L V-E F$ left ventricular ejection fraction

NYHA New York Heart Association

$R V O L$ regurgitant volume

$S P A P$ systolic pulmonary artery pressure

TAPSE tricuspid annular plane systolic excursion

to be severe during HG in only $5 \%$ of patients. Nonetheless, it should be mentioned that dynamic HG in patients with primary MR led to an increase in mean EROA from $18 \pm 9 \mathrm{~mm}^{2}$ to $22 \pm 11 \mathrm{~mm}^{2}$ (Table 1). This change led to a conversion of classification from mild to moderate MR in patients with primary disease. It should be emphasized, that there was small subgroup of patients with primary MR (5\%), who developed severe MR during HG (Table 5). In this subgroup baseline EROA was $34 \pm 5 \mathrm{~mm}^{2}$, which meant MR was already moderate at rest. These individuals were statistical outliers within the complete sample of patients with primary MR, who had a mean EROA of $18 \pm 9 \mathrm{~mm}^{2}$ at rest. However, as mentioned before, the role of exercise test in the evaluation of primary MR is not well established and should be further investigated.

A major constraint on the implementation of $\mathrm{HG}$ as a stress test in clinical routine might be the inconsistent intensity of contractions applied by different patients. The intensity of HG is usually measured as a percentage of the maximal voluntary contraction, which is a very subjective factor and is variable among patients [26-29]. Therefore, this intensity is expected not to be the same in the heterogeneous group of patients with MR. This may explain the variable effect of HG on MR in our study population.

It is common to withdraw beta-blockers before an exercise stress test. In our study population, $85 \%$ of patients were 


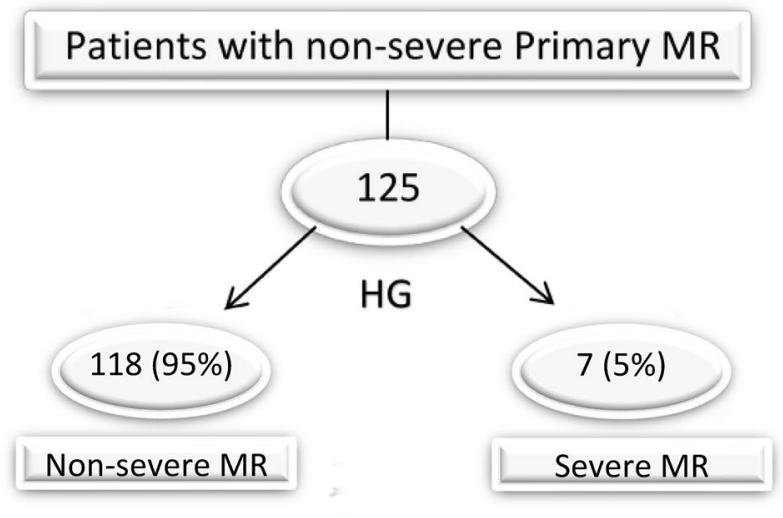

without beta-blocker $(n=69)$. A further study to evaluate the physiologic cardiovascular response to dynamic $\mathrm{HG}$ exercise in patients taking beta-blockers with and without withdrawing this medication may be of interest.

Conclusively, our study proves that dynamic HG, like dynamic exercise, increases MR to a variable extent in some patients and it therefore might be used in echocardiographic evaluation of MR, especially in symptomatic patients with moderate MR at rest.

\section{Limitations}

Although this is the first study to describe the effect of dynamic HG as a simple, bedside exercise method on MR in a large cohort of patients, we acknowledge that it has some limitations. The major limitation of this study is the lack of prognostic outcome data in patients with HG-induced increase in MR as well as the lack of the data comparing the effect of $\mathrm{HG}$ against the effect of dynamic exercise on the severity of MR. However, we believe that the results of this study may represent the basis for further research in this field. Another limitation is that classification of MR severity was only based on measurements of EROA and RVOL. Although quantification regurgitation severity using the PISA method remains the most recommended way to evaluate MR, this method may not be feasible in all patients. Furthermore, changes in blood pressure during HG were not reported. Finally, except for beta-blocker, the medical therapy of the patients was not described.

\section{Conclusions}

Fig. 3 Effect of handgrip (HG) exercise on the classification of regurgitation severity in primary and secondary mitral regurgitation (MR)

taking beta-blockers due to different indications. Although an increase in MR during HG was not significantly associated with a parallel increase in heart rate, as mentioned above, it cannot be ruled out that beta-blockers may have reduced the hemodynamic responses to HG and consequently its effect on the severity of MR. The finding, that intake of beta-blocker was associated with an upgrade in the classification of MR severity during HG in patients with secondary MR must be interpreted with caution. First, there was no significant association between intake of beta-blocker and the increase in MR during HG as shown in Tables 2 and 3. Secondly, in patients with secondary MR, there was a large difference in sample size between those with $(n=521)$ and

Dynamic HG exercise, as an easy to perform method during echocardiography, increased regurgitation severity in about half of patients with MR independent of the etiology. In patients with secondary MR, HG exercise led to a reclassification of regurgitation severity from non-severe $M R$ at rest to severe MR during HG in about one third of the patients. This effect was more evident in patients with exertional dyspnea and corresponded significantly to the NYHA dyspnea class. Therefore, dynamic HG may be used to identify exercise induced severe MR in symptomatic patients with moderate MR at rest. 
Table 4 Comparison of clinical characteristics and echocardiographic parameters between subgroups of patients with non-severe MR at rest and during HG and patients with non-severe MR at rest and severe MR during HG

\begin{tabular}{|c|c|c|c|c|c|c|}
\hline Variable & $\begin{array}{l}\text { Non-severe MR at } \\
\text { rest and during } \mathrm{HG}\end{array}$ & $\begin{array}{l}\text { Non-severe MR at rest } \\
\text { and severe MR during } \\
\text { HG }\end{array}$ & p-value & $\begin{array}{l}\text { Non-severe MR at } \\
\text { rest and during } \mathrm{HG}\end{array}$ & $\begin{array}{l}\text { Non-severe MR at rest } \\
\text { and severe MR during } \\
\text { HG }\end{array}$ & p-value \\
\hline Pathology & Primary & Primary & & secondary & secondary & \\
\hline Number of patients & 118 & 7 & & 271 & 104 & \\
\hline \multicolumn{7}{|l|}{ Clinical characteristics } \\
\hline Age, years & $68 \pm 17$ & $67 \pm 24$ & 0.934 & $73 \pm 11$ & $75 \pm 10$ & 0.272 \\
\hline Men, $\mathrm{n}$ & $37(32 \%)$ & $4(57 \%)$ & 0.158 & $134(49 \%)$ & $51(49 \%)$ & 0.944 \\
\hline Atrial fibrillation, $\mathrm{n}$ & $55(47 \%)$ & $4(57 \%)$ & 0.588 & $146(54 \%)$ & $64(61 \%)$ & 0.242 \\
\hline $\begin{array}{l}\text { NYHA functional classifi- } \\
\text { cation, } n\end{array}$ & & & 0.256 & & & 0.020 \\
\hline I & $54(47 \%)$ & $2(28 \%)$ & & $81(30 \%)$ & $17(16 \%)$ & \\
\hline II & $33(28 \%)$ & $1(14 \%)$ & & $90(33 \%)$ & $33(32 \%)$ & \\
\hline III & $26(22 \%)$ & $3(44 \%)$ & & $88(33 \%)$ & $49(47 \%)$ & \\
\hline IV & $4(3 \%)$ & $1(14 \%)$ & & $11(4 \%)$ & $5(5 \%)$ & \\
\hline Beta-blocker, $n$ & $80(68 \%)$ & $5(71 \%)$ & 0.854 & $232(86 \%)$ & $98(94 \%)$ & 0.026 \\
\hline $\begin{array}{l}\text { Presence of HR increase dur- } \\
\text { ing HG, } n\end{array}$ & $93(79 \%)$ & $5(71 \%)$ & 0.645 & $195(72 \%)$ & $75(72 \%)$ & 0.975 \\
\hline $\begin{array}{l}\text { Mean increase of HR during } \\
\mathrm{HG} \text {, (beat/min) }\end{array}$ & $7 \pm 8$ & $10 \pm 13$ & 0.357 & $6 \pm 7$ & $6 \pm 8$ & 0.902 \\
\hline \multicolumn{7}{|l|}{ Echocardiographic parameters } \\
\hline LV-EF, \% & $58 \pm 6$ & $60 \pm 2$ & 0.362 & $43 \pm 13$ & $40 \pm 12$ & 0.059 \\
\hline LA area, $\mathrm{cm}^{2}$ & $23 \pm 7$ & $26 \pm 7$ & 0.303 & $25 \pm 8$ & $25 \pm 7$ & 0.882 \\
\hline sPAP, $\mathrm{mmHg}$ & $32 \pm 12$ & $38 \pm 18$ & 0.433 & $35 \pm 12$ & $35 \pm 11$ & 0.799 \\
\hline TAPSE, mm & $21 \pm 5$ & $20 \pm 7$ & 0.807 & $19 \pm 6$ & $19 \pm 5$ & 0.211 \\
\hline EROA at rest, $\mathrm{mm}^{2}$ & $15 \pm 6$ & $34 \pm 5$ & $<0.001$ & $12 \pm 4$ & $15 \pm 2$ & $<0.001$ \\
\hline EROA during $\mathrm{HG}, \mathrm{mm}^{2}$ & $18 \pm 7$ & $51 \pm 9$ & $<0.001$ & $14 \pm 4$ & $23 \pm 4$ & $<0.001$ \\
\hline $\mathrm{RVOL}$ at rest, $\mathrm{ml}$ & $28 \pm 11$ & $52 \pm 10$ & $<0.001$ & $22 \pm 8$ & $26 \pm 6$ & $<0.001$ \\
\hline RVOL during $\mathrm{HG}, \mathrm{ml}$ & $34 \pm 14$ & $74 \pm 15$ & $<0.001$ & $26 \pm 8$ & $39 \pm 9$ & $<0.001$ \\
\hline LVEDd, mm & $48 \pm 6$ & $54 \pm 7$ & 0.231 & $54 \pm 10$ & $52 \pm 11$ & 0.850 \\
\hline LVEDd, mm & $32 \pm 7$ & $37 \pm 3$ & 0.129 & $44 \pm 12$ & $43 \pm 2$ & 0.473 \\
\hline
\end{tabular}

EROA effective regurgitant orifice area

$L A$ area left atrial area

LVEDd left ventricular end-diastolic diameter

$L V E D$ s left ventricular end-systolic diameter

$L V$-EF left ventricular ejection fraction

NYHA New York Heart Association,

RVOL regurgitant volume,

$S P A P$ systolic pulmonary artery pressure

TAPSE tricuspid annular plane systolic excursion 
Table 5 Association between clinical variables and echocardiographic parameters and the upgrade in MR severity calssification from non-severe MR at rest to severe MR during HG in patients with primary and secondary MR

\begin{tabular}{|c|c|c|c|c|}
\hline \multirow[t]{2}{*}{ Variable } & \multicolumn{2}{|l|}{ Primary MR } & \multicolumn{2}{|l|}{ Secondary MR } \\
\hline & OR, $95 \%$-CI & p-value & OR, $95 \%$-CI & p-value \\
\hline \multicolumn{5}{|l|}{ Clinical variables } \\
\hline Age (per year) & $0.998(0.955-1.043)$ & 0.934 & $1.012(0.991-1.034)$ & 0.272 \\
\hline Gender (Men) & $0.343(0.073-1.609)$ & 0.175 & $1.034(0.651-1.610)$ & 0.918 \\
\hline Atrial fibrillation & $1.527(0.327-7.124)$ & 0.590 & $1.316(0.830-2.084)$ & 0.243 \\
\hline NYHA functional Classification (per class) & $1.947(0.872-4.341)$ & 0.104 & $1.486(1.138-1.940)$ & 0.004 \\
\hline NT-proBNP (per 100 pg/nl) & $1.007(0.821-1.236)$ & 0.947 & $1.070(0.992-1.153)$ & 0.078 \\
\hline Increase of heart rate during $\mathrm{HG}$ & $0.672(0.123-3.672)$ & 0.646 & $1.008(0.609-1.668)$ & 0.975 \\
\hline Beta-blocker & $1.187(0.220-6.401)$ & 0.842 & $2.675(1.096-6.533)$ & 0.031 \\
\hline \multicolumn{5}{|l|}{ Echocardiographic parameters } \\
\hline EROA at rest (per $\mathrm{mm}^{2}$ ) & $2.485(0.999-6.184)$ & 0.050 & $1.308(1.209-1.415)$ & $<0.001$ \\
\hline RVOL at rest (per ml) & $1.239(1.090-1.409)$ & 0.001 & $1.065(1.032-1.100)$ & $<0.001$ \\
\hline LV-EF & $1.277(0.790-2.066)$ & 0.318 & $0.983(0.966-1.001)$ & 0.060 \\
\hline LA area $\left(\right.$ per $\left.\mathrm{cm}^{2}\right)$ & $1.055(0.952-1.170)$ & 0.303 & $1.002(0.973-1.032)$ & 0.881 \\
\hline sPAP (per mmHg) & $1.033(0.979-1.090)$ & 0.235 & $0.798(0.983-1.023)$ & 0.798 \\
\hline TAPSE (per mm) & $0.980(0.834-1.151)$ & 0.805 & $0.970(0.925-1.017)$ & 0.210 \\
\hline LVEDd (per mm) & $1.161(1.015-1.328)$ & 0.209 & $0.998(0.976-1.020)$ & 0.849 \\
\hline LVEDs (per mm) & $1.098(0.975-1.217)$ & 0.131 & $9.996(0.977-1.014)$ & 0.635 \\
\hline
\end{tabular}

EROA effective regurgitant orifice area

$L A$ area left atrial area

LVEDd left ventricular end-diastolic diameter

$L V E D s$ left ventricular end-systolic diameter

$L V$-EF left ventricular ejection fraction

NYHA New York Heart Association

$R V O L$ regurgitant volume

SPAP systolic pulmonary artery pressure

TAPSE tricuspid annular plane systolic excursion

\begin{abstract}
Author contributions MNA wrote the paper and has made substantial contributions to the acquisition, analysis and interpretation of data. AK has made substantial contributions to the acquisition, analysis and interpretation of data. JG has made substantial contributions to the analysis and interpretation of data. MA has made substantial contributions to the analysis and interpretation of data. MF has made substantial contributions to the analysis and interpretation of data. AM has made substantial contributions to the analysis of data. WM has made substantial contributions to the acquisition and interpretation of data. MB has made substantial contributions to the conception and design of the work and interpretation of data. NM has made substantial contributions to the interpretation of data and has substantively revised the work. EA has made substantial contributions to the conception and design of the work, to the analysis and interpretation of data and has substantively revised the work.
\end{abstract}

Funding Open Access funding enabled and organized by Projekt DEAL.

\section{Compliance with ethical standards}

Conflict of interest The authors declare that they have no conflict of interest.

Open Access This article is licensed under a Creative Commons Attribution 4.0 International License, which permits use, sharing, adaptation, distribution and reproduction in any medium or format, as long as you give appropriate credit to the original author(s) and the source, provide a link to the Creative Commons licence, and indicate if changes were made. The images or other third party material in this article are included in the article's Creative Commons licence, unless indicated otherwise in a credit line to the material. If material is not included in the article's Creative Commons licence and your intended use is not permitted by statutory regulation or exceeds the permitted use, you will need to obtain permission directly from the copyright holder. To view a copy of this licence, visit http://creativecommons.org/licenses/by/4.0/. 


\section{References}

1. McCraw DB, Siegel W, Stonecipher HK, Nutter DO, Schlant RC, Hurst JW (1972) Response of heart murmur intensity to isometric (handgrip) exercise. Br Heart J 34(6):605-610

2. Dattilo G, Patanè S, Zito C, Lamari A, Tulino D, Marte F, Carerj S (2010) Handgrip exercise associated with dobutamine stress echocardiography. Int J Cardiol 143(3):298-301

3. Jake Samuel T, Beaudry R, Haykowsky MJ, Sarma S, Park S, Dombrowsky T, Bhella PS, Nelson MD (2017) Isometric handgrip echocardiography: a noninvasive stress test to assess left ventricular diastolic function. Clin Cardiol 40(12):1247-1255

4. Velu JF, Baan J Jr, de Bruin-Bon HACM et al (2019) Can stress echocardiography identify patients who will benefit from percutaneous mitral valve repair? Int J Cardiovasc Imaging 35(4):645-651

5. Nkomo VT, Gardin JM, Skelton TN, Gottdiener JS, Scott CG, Enriquez-Sarano M (2006) Burden of valvular heart diseases: a population-based study. Lancet 368(9540):1005-1011

6. Enriquez-Sarano M, Akins CW, Vahanian A (2009) Mitral regurgitation. Lancet 373(9672):1382-1394

7. Rossi A, Dini FL, Faggiano P, Agricola E, Cicoira M, Frattini S, Simioniuc A, Gullace M, Ghio S, Enriquez-Sarano M, Temporelli PL (2011) Independent prognostic value of functional mitral regurgitation in patients with heart failure. A quantitative analysis of 1256 patients with ischaemic and non-ischaemic dilated cardiomyopathy. Heart 97(20):1675-1680

8. Boekstegers P, Hausleiter J, Baldus S, von Bardeleben RS, Beucher H, Butter C, Franzen O, Hoffmann R, Ince H, Kuck KH, Rudolph V, Schäfer U, Schillinger W, Wunderlich N (2014) Percutaneous interventional mitral regurgitation treatment using the Mitra-Clip system. Clin Res Cardiol 103(2):85-96

9. Ansari MT, Ahmadzai N, Coyle K, Coyle D, Moher D (2015) Mitral valve clip for treatment of mitral regurgitation: an evidencebased analysis. Ont Health Technol Assess Ser 15(12):1-104

10. Lancellotti P, Garbi M (2018) Exercise stress echocardiography in degenerative mitral regurgitation. Circ Cardiovasc Imaging 11(9):e008263

11. Dulgheru R, Marchetta S, Sugimoto T, Go YY, Girbea A, Oury C, Lancelotti P (2017) Exercise testing in mitral regurgitation. Prog Cardiovasc Dis 60(3):342-350

12. Lancellotti P, Gérard PL, Piérard LA (2005) Long-term outcome of patients with heart failure and dynamic functional mitral regurgitation. Eur Heart J 26(15):1528-1532

13. Salden OAE, van Everdingen WM, Spee R, Doevendans PA, Cramer MJ (2018) How I do it: feasibility of a new ultrasound probe fixator to facilitate high quality stress echocardiography. Cardiovasc Ultrasound 16(1):6

14. Peteiro J, Bouzas-Mosquera A, Estevez R, Pazos P, Piñeiro M, Castro-Beiras A (2012) Head-to-head comparison of peak supine bicycle exercise echocardiography and treadmill exercise echocardiography at peak and at post-exercise for the detection of coronary artery disease. J Am Soc Echocardiogr 25(3):319-326

15. Evangelista A, Flachskampf F, Lancellotti P, Badano L, Aguilar R, Monaghan M et al (2008) European Association of Echocardiography recommendations for standardization of performance, digital storage and reporting of echocardiographic studies. Eur J Echocardiogr 9(4):438-448

16. Lancellotti P, Tribouilloy C, Hagendorff A, Popescu BA, Edvardsen T, Pierard LA, Zamoramo JL (2013) Recommendations for the echocardiographic assessment of native valvular regurgitation: an executive summary from the European Association of Cardiovascular Imaging. Eur Heart J Cardiovasc Imaging 14(7):611-644
17. Hill SA, Booth RA, Santaguida PL, Don-Wauchope A, Brown JA, Oremus M, Ali U, Sohel N, McKelvie R, Balion C, Raina P (2014) Use of BNP and NT-proBNP for the diagnosis of heart failure in the emergency department: a systematic review of the evidence. Heart Fail Rev 19(4):421-438

18. Maries L, Manitiu I (2013) Diagnostic and prognostic values of B-type natriuretic peptides (BNP) and $\mathrm{N}$-terminal fragment brain natriuretic peptides (NT-pro-BNP). Cardiovasc J Afr 24(7):286-289

19. Dal-Bianco JP, Beaudoin J, Handschumacher MD, Levine RA (2014) Basic mechanisms of mitral regurgitation. Can J Cardiol 30(9):971-981

20. Baumgartner H, Falk V, Bax JJ, De Bonis M, Hamm C, Holm PJ, Lung B, Lancelotti P, Lansac E, Mu noz DR, Rosenhek R, Sjögren J, Tornos Mas P, Vahanian A, Walther T, Wendler O, Windecker S, Luis Zamorano J, (2017) 2017 ESC/EACTS Guidelines for the management of valvular heart disease. Eur Heart J 38(36):2739-2791

21. Laughlin MH (1999) Cardiovascular response to exercise. Am J Physiol 277(6 Pt 2):S244-S259

22. Lind AR, McNicol GW (1967a) Muscular factors which determine the cardiovascular responses to sustained and rhythmic exercise. Can Med Assoc J 96(12):706-715

23. Motoi Y, Forton K, Pezzuto B, Fauro V, Naeiji R (2017) Resistive or dynamic exercise stress testing of the pulmonary circulation and the right heart. Eur Respir J 50:1700151

24. Louhevaara V, Smolander J, Aminoff T et al (2000) Cardiorespiratory responses to fatiguing dynamic and isometric hand-grip exercise. Eur J Appl 82(4):340-344

25. Lind AR, McNicol GW (1967b) Circulatory responses to sustained hand-grip contractions performed during other exercise, both rhythmic and static. J Physiol 192(3):595-607

26. Picano E, Pibarot P, Lancellotti P, Monin JL, Bonow RO (2009) The emerging role of exercise testing and stress echocardiography in valvular heart disease. J Am Coll Cardiol 54(24):2251-2260

27. Bertrand PB, Schwammenthal E, Levine RA, Vandervoort PM (2017) Exercise dynamics in secondary mitral regurgitation: pathophysiology and therapeutic implications. Circulation 135(3):297-314

28. Keren G, Katz S, Gage J, Strom J, Sonnenblick EH, LeJemtel TH (1989) Effect of isometric exercise on cardiac performance and mitral regurgitation in patients with severe congestive heart failure. Am Heart J 118(5 Pt 1):973-979

29. Beyer SE, Sanghvi MM, Aung N, Hosking A, Cooper JA, Paiva JM, LEE AK., Fung K, Lujaschuk E, Carapella V, Mittelman MA., Brage S, Piechnik SK., Neubauer S, Petersen SE, (2018) Prospective association between handgrip strength and cardiac structure and function in UK adults. PLoS ONE 13(3):e0193124

30. Hartog R, Bolignano D, Sijbrands E, Pucci G, Mattace-Raso F (2018) Short-term vascular hemodynamic responses to isometric exercise in young adults and in the elderly. Clin Interv Aging 13:509-514

31. Lalande S, Sawicki CP, Baker JR, Shoemaker JK (2014) Effect of age on the hemodynamic and sympathetic responses at the onset of isometric handgrip exercise. J Appl Physiol (1985) 116(2):222-227

32. Grucza R, Smorawiński J, Cybulski G, Niewiadomski W, Kahn JF, Kapitaniak BMonod H. (1991) Cardiovascular response to static handgrip in trained and untrained men. Eur J Appl Physiol Occup Physiol 62(5):337-341

Publisher's Note Springer Nature remains neutral with regard to jurisdictional claims in published maps and institutional affiliations. 